\title{
SHORT TIME RESULT OF PERCUTANEOUS TRANSLUMINAL ANGIOPLASTY ON ISCHEMIA DIABETIC FOOT ULCER
}

\author{
HENDRY IRAWAN ${ }^{1 *}$, DANNY ZEFANYA MOOY ${ }^{1}$, KETUT PUTU YASA ${ }^{2}$
}

${ }^{1}$ Department of Surgery, Faculty of Medicine Udayana University, Sanglah General Hospital, Bali, Indonesia. ${ }^{2}$ Department of Cardiothoracic and Vascular Surgery, Faculty of Medicine Udayana University, Sanglah General Hospital, Bali, Indonesia.

Email: hendry_irawan@rocketmail.com

Received: 15 January 2018, Revised and Accepted: 09 February $2018^{</ A Q 1>}$

ABSTRACT

The frequent complication of diabetes mellitus is ischemic diabetic foot ulcers (DFUs). As compared to non-diabetic people, ischemic of foot prevalence is significantly higher in diabetic people. It is related with severe clinical manifestations and the risk of critical limb ischemia and limb loss is increasing. In this case, we performed percutaneous transluminal angioplasty (PTA) in patient with ischemic DFU. A male, 58-year old, complained severe rest pain and gangrene in the fourth toe of the left foot more than 2 months. He had a history of diabetes mellitus type 2 for 4 years ago with uncontrolled blood sugar. We perform PTA to decrease the rest pain and prevent expand of the gangrene. The result of PTA was shown instant effect to reduce rest pain, pulses are easily palpable, and there was no expansion of the gangrene.

Keywords: Ischemic diabetic foot ulcer, Percutaneous transluminal angioplasty, Rest pain.

(C) 2018 The Authors. Published by Innovare Academic Sciences Pvt Ltd. This is an open access article under the CC BY license (http://creativecommons. org/licenses/by/4. 0/) DOI: http://dx.doi.org/10.22159/ajpcr.2018.v11i5.24759

\section{INTRODUCTION}

Approximately $5 \%$ of diabetic patients have a history of diabetic foot ulcers (DFUs) and 15\% experience the complication during their lifetime. The most complication of the DFUs is amputation [1]. The most frequent cause of amputation is ischemia and infection [1-3]. Vasculopathy and neuropathy have a combination role to cause DFUs. It can lead neuroischemic ulcers that can decrease oxygen supply to distal foot [4,5]. Ischemia caused by peripheral arterial disease (PAD) and it is more frequent in patients with diabetes, and approximately up to $50 \%$ of patients with DFUs have PAD [2,3]. The mortality of DFU patients with PAD is high, $50 \%$ of patients' dead at 5 years, and the results are worse after major amputation with $50 \%$ mortality after 2 years [3].

The successful revascularization can reduce rate of amputation. The effectiveness of peripheral bypass and percutaneous transluminal angioplasty (PTA) had been reported $[1,6]$. PTA was performed with digital fluoroscopy guidance and evaluation of lower leg. That procedure can open stenosis or occlusion vessels using balloon dilatation catheter and restore blood flow to distal vessels [7]. The patient with occlusion involved intermittent claudication, cramp-like pain that occurs during exercise and relieved with rest, or ischemic rest pain during the night, especially on ischemic ulcer or gangrenous toe [1]

In our institution, revascularization usually does with open bypass surgery, but fortunately there is new facility of catheterization laboratory and endovascular therapy, so we do the other method of revascularization to treat limb ischemia. We reported ischemic DFU patient with total occlusion of left tibial artery. He had good outcome after PTA to relieve rest pain, improved distal circulation of limb, and prevent expand of the gangrene.

\section{CASE REPORT}

A male, 58-year old, complained severe pain and wound of his left foot for 2 months ago. He just controlled in local health care near his house. The pain is getting worse and the wound in the fourth toe of the left foot becomes necrotic and surrounding skin was blackish. He had a history of diabetes mellitus type 2 for 4 years ago with uncontrolled blood sugar and did not routine use drugs. The physical examination of his left foot revealed necrotic tissue of fourth toe, discharge of pus, exudates, and bad odor (Fig. 1). Distal vascular perfusion was not good, and it is hard to palpable popliteal artery and tibialis anterior artery. The radiograph of the left foot results osteomyelitis with no gas gangrene (Fig. 2).

We performed arteriography first to diagnose the problem with the vascular. Patient's angiograms of the iliac, femoral, popliteal, peroneus, and tibial arteries of the ulcerated side were obtained. We got the total occlusion of left tibial artery with not enough contras flow to foot (Fig. 3).

After that we performed PTA to open the blocked in left tibial artery. We observe the rest pain and revascularization after PTA (Fig. 4). The condition was better, decreasing rest pain, and distal pulsation of artery was palpable. However, the gangrenous fourth toe was irreversible, at the end, his fourth toe was amputated at next 6 months. After 1-year follow-up, there was no expansion of the gangrene to other toes, complete wound healing, and good pulsation of distal vessels.

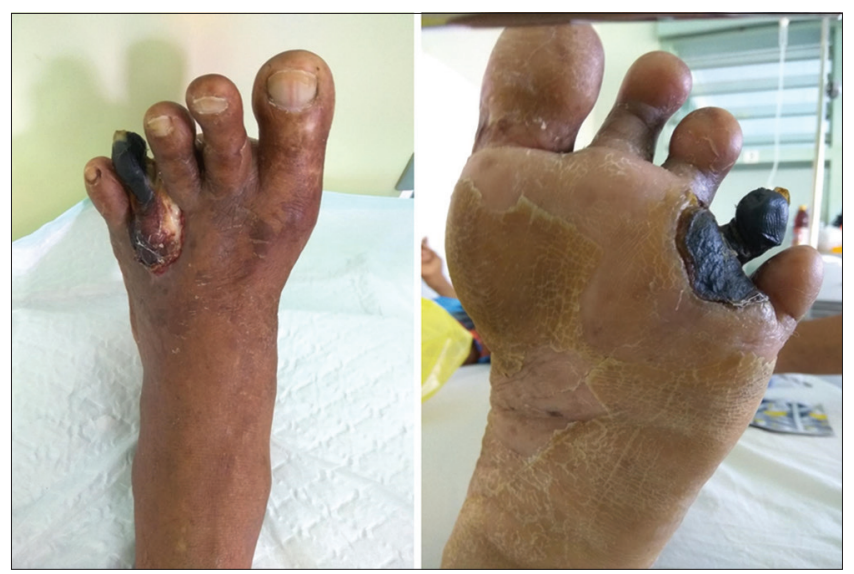

Fig. 1: Clinical picture when the patient comes to hospital 


\section{DISCUSSION}

Diabetic patients with ulcer have poor healing wound, neuropathy, and peripheral vascular disease [5]. Lower limb PAD is a frequent complication of diabetes mellitus, and the prevalence is significantly higher in diabetic patients. Lower limb PAD can cause severe morbidity such as critical limb ischemia (CLI) and lower limb loss [8]. In diabetic people, the poor outcome of ischemic foot ulcers is probably linked to multifactor, including the location of the vascular injury, the association
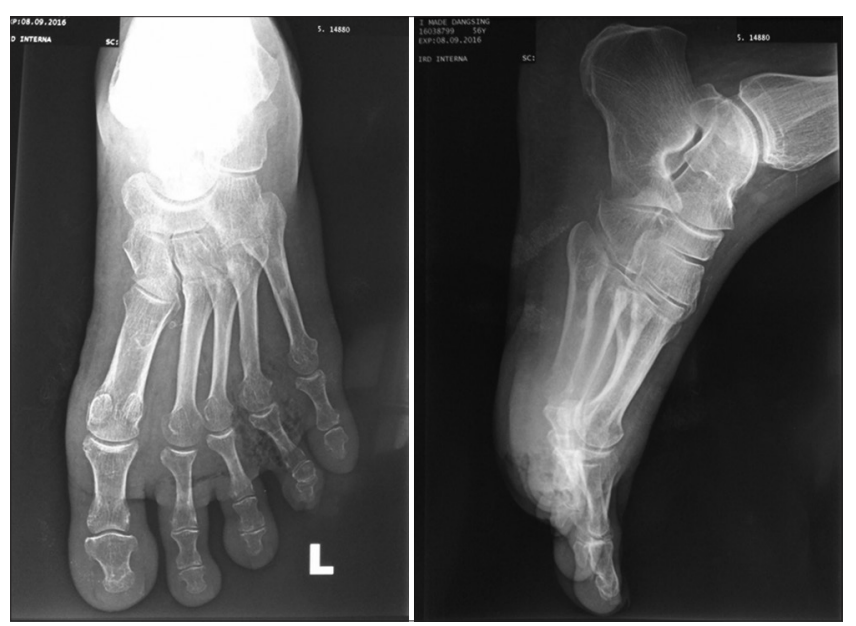

Fig. 2: Radiograph of the left foot

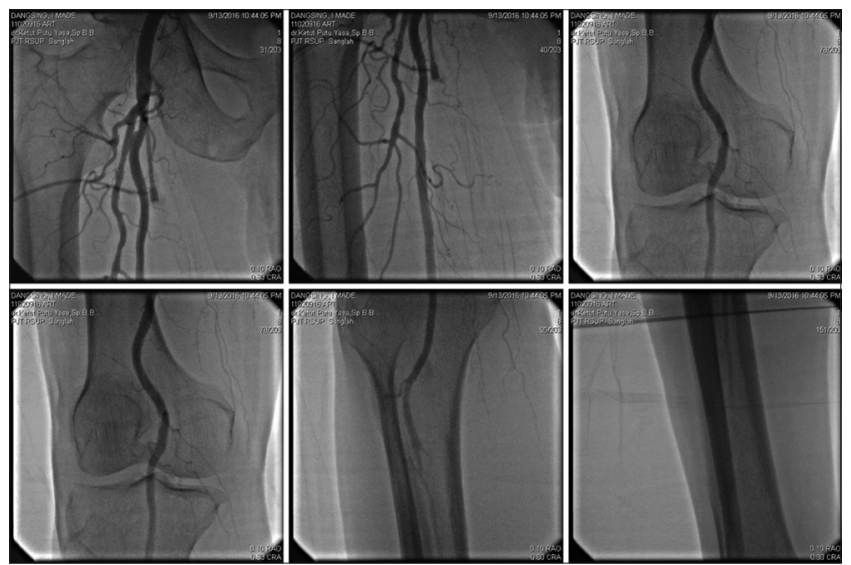

Fig. 3: Arteriography, total occlusion of left tibial artery with other disease (such as neuropathy, infection, and renal failure), and the presence of complication in other vascular area (such as coronary or cerebral arteries) [3].

In diabetic patients, pure ischemic ulcers are less frequent and the vast majority of ulcers are either pure neuropathy or mixed neuroischemia [1]. In diabetic people with ischemic DFU, successful revascularization can reduce the rate of major amputation [6]. CLI is defined by the presence of ischemic skin lesions (ulcers or gangrene) and symptoms of rest pain of more than 2 weeks that require regular analgesics. Ischemic rest pain most typically occurs at night (when the limb is no longer in a dependent position) but in severe cases can be continuous [1].

Active revascularization plays an important role in achieving ulcer healing, and over the past decade, the endovascular therapy was used to DFUs patients [9]. The endovascular therapy of stenosis and occlusion of the superficial femoral artery remains one of the biggest challenges in modern vascular practice. The length of the stenosis or occlusion is the most important indicator of success of PTA [1].

The outcome of both open surgery and endovascular therapy is the same for ulcer healing and limb salvage, between $85 \%$ and $78 \%[3,9]$. Other study, after 1-year follow-up, more than $60 \%$ of ulcers had healed following revascularization with either open bypass surgery or endovascular revascularization [3]. Endovascular therapy can be easily repeated, is well tolerated by the patient, and is associated with low rates of serious complications [1].

In one study of diabetic foot patients with severe ischemia, the rate of clinical success after PTA (it is defined as partial or total ulcer healing with no bypass grafting or major amputation) was $52 \%$ [7]. The overall 1 -year limb salvage rate in patients with CLI was $30 \%$ to $50 \%$ of diabetic patients [10], and it shows that diabetes does not necessarily indicate a poor prognosis [7].

The studies demonstrated improved rates of limb salvage associated with revascularization to treat patients with DFUs [3]. The patient with CLI is the indication of revascularization and it can improve distal flow. Base on society for vascular surgery practice guidelines, both endovascular and surgical therapy, can improve patients' quality of life, reduce pain, relieve symptoms of claudication, and improve ambulatory function [11].

The efficacy of both therapies was similar [11,12], but endovascular surgery will give quick response, minimal invasive, and high successful rate like use open surgery [12]. To our opinion, endovascular therapy is very appealing in such cases, and we would consider this as a first

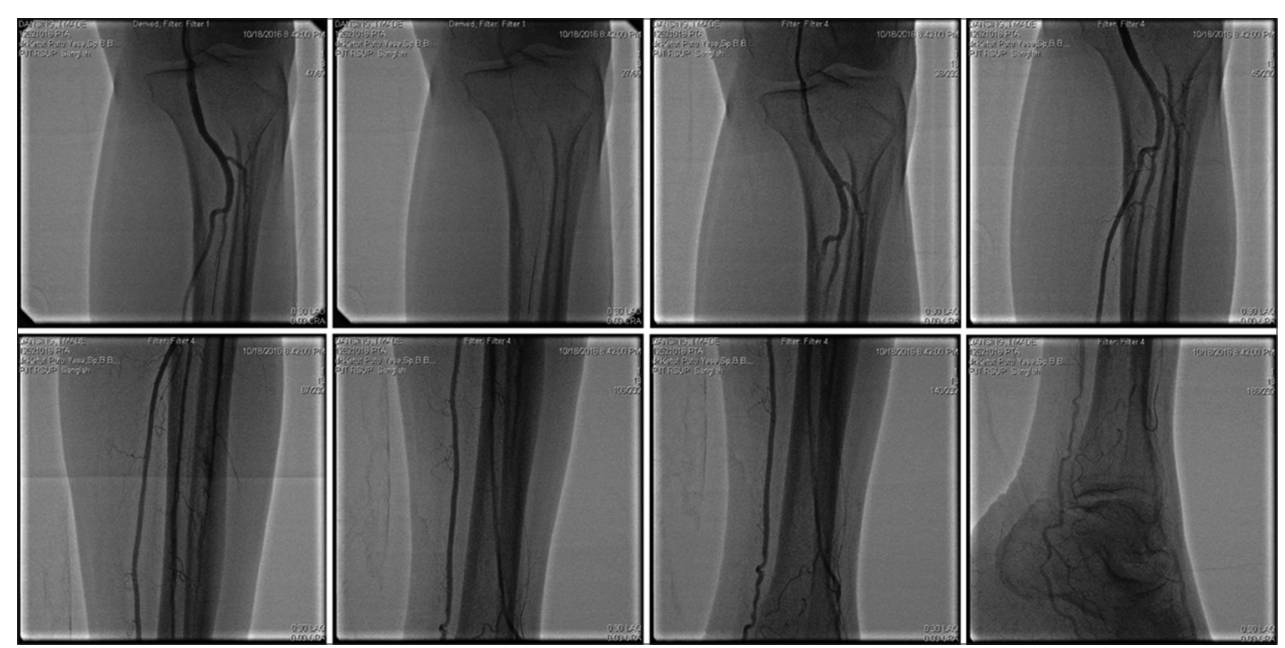

Fig. 4: Percutaneous transluminal angioplasty 
option. Transluminal angioplasty certainly has its place and may be useful for stenotic or occlusive lesions and give instant result to decrease rest pain. In short time result, PTA gives more advantages over open bypass surgery.

The management of the diabetic foot needs a multidisciplinary approach. Once ischemic ulceration has developed, aggressive management can achieve excellent results with a significant reduction of amputation and reulceration rates similar to those seen in non-diabetics. A recipe for success includes a combination of revascularization, surgical debridement, and appropriate antibiotics.

The other factors that can help improving good outcome are healthy food, especially low sugar food, doing physical activity, blood sugar control, and compliance with medications [5].

\section{CONCLUSION}

Ischemia caused by PAD and is more frequent in patients with DFUs. That complication of DFUs can lead to rest pain, non-healing ulcer, and limb loss. The result of PTA was shown instant effect to reduce rest pain, pulses are easily palpable, and there was no expansion of the gangrene.

\section{AUTHORS CONTRIBUTION}

All authors worked to do this manuscript. Hendry Irawan collected data, wrote final manuscript, and editing of manuscript, Danny Zefanya Mooy wrote first draft and collected data, and Ketut Putu Yasa designed the study and performed arteriography and PTA. All authors read and approved the final manuscript.

\section{CONFLICT OF INTERESTS}

All the authors have no conflict of interest.

\section{REFERENCES}

1. Gerassimidis T, Karkos CD, Karamanos D, Kamparoudis A. Current endovascular management of the ischaemic diabetic foot. Hippokratia 2008;12:67-73.
2. Hinchliffe RJ, Brownrigg JR, Apelqvist J, Boyko EJ, Fitridge R, Mills JL, et al. IWGDF guidance on the diagnosis, prognosis and management of peripheral artery disease in patients with foot ulcers in diabetes. Diabetes Metab Res Rev 2016;32:37-44.

3. Hinchliffe RJ, Andros G, Apelqvist J, Bakker K, Friederichs S, Lammer J, et al. A systematic review of the effectiveness of revascularization of the ulcerated foot in patients with diabetes and peripheral arterial disease. Diabetes Metab Res Rev 2012;28 Suppl 1:179-217.

4. Karri VV. Current perspective in the management of diabetic foot ulcers - An overview on the Indian scenario. Int J Pharm Pharm Sci 2014;6:1-2.

5. Thomas SM, Nitin IG, Reddy MU, Devi H. A prospective study: Knowledge assessment and patient care of diabetic foot ulcer patients in tertiary care hospital. Int J Pharm Pharm Sci 2017;9:104-10.

6. Faglia E, Mantero M, Caminiti M, Caravaggi C, De Giglio R, Pritelli C, et al. Extensive use of peripheral angioplasty, particularly in frapopliteal, in the treatment of ischaemic diabetic foot ulcers: Clinical results of a multicentric study of 221 consecutive diabetic subjects. J Intern Med 2002;252:225-32.

7. Jacqueminet S, Hartemann-Heurtier A, Izzillo R, Cluzel P, Golmard JL, Ha Van $\mathrm{G}$, et al. Percutaneous transluminal angioplasty in severe diabetic foot ischemia: Outcomes and prognostic factors. Diabetes Metab 2005;31:370-5.

8. Graziani L, Silvestro A, Bertone V, Manara E, Andreini R, Sigala A, et al. Vascular involvement in diabetic subjects with ischemic foot ulcer: A new morphologic categorization of disease severity. Eur J Vasc Endovasc Surg 2007;33:453-60.

9. Reekers JA, Lammer J. Diabetic foot and PAD: The endovascular approach. Diabetes Metab Res Rev 2012;28:36-9.

10. Nasr MK, McCarthy RJ, Hardman J, Chalmers A, Horrocks M. The increasing role of percutaneous transluminal angioplasty in the primary management of critical limb ischaemia. Eur J Vasc Endovasc Surg 2002;23:398-403.

11. Conte MS, Pomposelli FB, Clair DG, Geraghty PJ, McKinsey JF, Mills JL, et al. Society for vascular surgery practice guidelines for atherosclerotic occlusive disease of the lower extremities: Management of asymptomatic disease and claudication. J Vasc Surg 2015:61:2S-41S.

12. Bradbury AW, Adam DJ, Bell J, Forbes JF, Fowkes FG, Gillespie I, et al. Bypass versus angioplasty in severe ischaemia of the leg (BASIL) trial: A survival prediction model to facilitate clinical decision making. J Vasc Surg 2010;51:52S-68S. 